\title{
1 Bivariate genomic prediction of phenotypes by selecting epistatic interactions 2 across years
}

\author{
3 Elaheh Vojgani ${ }^{1 *}$, Torsten Pook ${ }^{1}$, Armin C. Hölker ${ }^{2}$, Manfred Mayer ${ }^{2}$, Chris-Carolin Schön ${ }^{2}$, \\ 4 Henner Simianer ${ }^{1}$ \\ $5 \quad{ }^{1}$ University of Goettingen, Center for Integrated Breeding Research, Animal Breeding and \\ 6 Genetics Group, Goettingen, Germany; ${ }^{2}$ Plant Breeding, TUM School of Life Sciences \\ 7 Weihenstephan, Technical University of Munich, Freising, Germany
}

9 Key Massage

10 Bivariate models based on selected subsets of pairwise SNP interactions can increase the 11 prediction accuracy by utilizing phenotypic data across years under the assumption of high 12 genomic correlation across years.

13 Abstract

14 The importance of accurate genomic prediction of phenotypes in plant breeding is undeniable, 15 as higher prediction accuracy can increase selection responses. In this study, we investigated the 16 ability of three models to improve prediction accuracy by including phenotypic information from 17 the last growing season. This was done by considering a single biological trait in two growing 18 seasons (2017 and 2018) as separate traits in a multi-trait model. Thus, bivariate variants of the 19 Genomic Best Linear Unbiased Prediction (GBLUP) as an additive model, Epistatic Random Regression BLUP (ERRBLUP) and selective Epistatic Random Regression BLUP (sERRBLUP) as epistasis models were compared with respect to their prediction accuracies for the second year. The results indicate that bivariate ERRBLUP is slightly superior to bivariate GBLUP in predication accuracy, while bivariate SERRBLUP has the highest prediction accuracy in most cases. The average relative increase in prediction accuracy from bivariate GBLUP to maximum bivariate sERRBLUP across eight phenotypic traits and studied dataset from 471/402 doubled haploid lines in the European maize landrace Kemater Landmais Gelb/Petkuser Ferdinand Rot, were 7.61 and 3.47 percent, respectively. We further investigated the genomic correlation, phenotypic correlation and trait heritability as the factors affecting the bivariate model's predication accuracy, with genetic correlation between growing seasons being the most important one. For all three considered model architectures results were far worse when using a univariate version of the model, e.g. with an average reduction in prediction accuracy of $0.23 / 0.14$ for Kemater/Petkuser when using univariate GBLUP.

\section{Keywords:}

34 Epistasis, Bivariate GBLUP, Prediction across years, Genomic correlation 
Introduction

In plant breeding, genomic prediction has become a daily tool (Bernal-Vasquez et al. 2014; Stich and Ingheland 2018) which enables the optimization of phenotyping costs of breeding programs (Akdemir and Isidro-Sánchez 2019). The importance of genomic prediction of phenotypes is not restricted to plants. Livestock (Daetwyler et al. 2013) and human research (de los Campos et al. 2013) also have been widely developed in this regard. In the context of plant and animal breeding, accurately predicting phenotypic traits is of special importance, since raising all animals and growing all crops to measure their performances requires a considerable amount of money under limited resources (Martini et al. 2016).

Several statistical models have been compared over the last decades in the term of prediction accuracy. In this context, genomic best linear unbiased prediction (GBLUP) (Meuwissen et al. 2001; VanRaden 2007) as an additive linear mixed model has been widely used due to its high robustness, computing speed and superiority in predictive ability to alternative prediction models like Bayesian methods, especially in small reference populations ( $D a$ et al. 2014; Rönnegård and Shen 2016; Covarrubias-Pazaran et al. 2018; Wang et al. 2018). Furthermore, inclusion of genotype $\times$ environment interaction into additive genomic prediction models can result in an increase in prediction accuracy (Hallauer et al. 2010; Bajgain et al. 2020). Such approaches allow borrowing information across environments which potentially leads to higher accuracy in phenotype prediction in multi environment models (Burgueño et al. 2012). In fact, multivariate mixed models have been originally proposed in the context of animal breeding (Henderson and Quaas 1976) with the purpose of modeling the genomic correlation among traits, longitudinal data, and modeling genotype by environment interactions across multiple years or environments (Mrode 2014; Lee and van der Werf 2016; Covarrubias-Pazaran et al. 2018). A multivariate GBLUP model was reported to have higher prediction accuracy than univariate GBLUP (Jia and Jannink 2012) when the genetic correlations were medium (0.6) or high (0.9) (Covarrubias-Pazaran et al. 2018). It was also shown that aggregating the phenotypic data over years to train the model and predict the performance of lines in the following years is a possible approach which can improve prediction accuracy (Auinger et al. 2016; Schrag et al. 2019a).

In addition, inclusion of epistasis, defined as the interaction between loci (Falconer and Mackay 1996; Lynch and Walsh 1998), into the genomic prediction model results in more accurate phenotype prediction (Hu et al. 2011; Wang et al. 2012; Mackay 2014; Martini et al. 2016; Vojgani et al. 2019b) due to the considerable contribution of epistasis in genetic variation of quantitative traits (Mackay 2014). In this context, several statistical models have been proposed. Extended genomic best linear unbiased prediction (EG-BLUP, Jiang and Reif 2015) and categorical epistasis (CE, Martini et al. 2017) models are using a marker-based epistatic relationship matrix that is constructed in a highly efficient manner. It has been shown that the CE model is as good as or better than EG-BLUP and does not possess undesirable features of EG-BLUP such as codingdependency (Martini et al. 2017). 
Moreover, it was shown that the accuracy of the epistasis genomic prediction model can be increased in one environment by variable selection in another environment (Martini et al. 2016). In this approach, the full epistasis model was reduced to a model with a subset of the largest epistatic interaction effects, resulting in an increase in predictive ability (Martini et al. 2016), through borrowing information across environments. Vojgani et al. (2019b) showed that the prediction accuracy can be increased even further by selecting the interactions with the highest absolute effect sizes / variances in the epistasis model. Resulting higher computational needs were offset by the development of a highly efficient software package (Vojgani et al. 2019a) to perform computations in a bit-wise manner (Schlather 2020). Thus, enabling to conduct such predictions with data sets of practically relevant size across environments in the same year, both with respect to sample size and number of markers (Vojgani et al. 2019b).

The aim of this study is to assess the bivariate genomic prediction models which incorporate pairwise SNP interactions with the target of borrowing information across years to maximize the predictive ability. Since the accuracy of genomic prediction of phenotypes was shown to be increased by both borrowing information across environments and years (Covarrubias-Pazaran et al. 2018; Schrag et al. 2019b) and inclusion of epistasis into the prediction model (Martini et al. 2016; Vojgani et al. 2020), we combine these two approaches to make the best use of the available information. We further aim to assess the optimum proportion of SNP interactions to be kept in the model in the variable selection step across years. The data used for this purpose were generated in multi-location trials of doubled haploid (DH) lines generated from two European maize landraces in 2017 and 2018.

Materials and Methods

\section{Data used for analysis}

A set of 948 doubled haploid lines of the European maize landraces Kemater Landmais Gelb (KE, Austria, 516 lines) and Petkuser Ferdinand Rot (PE, Germany, 432 lines) were genotyped with the 600 k Affymetrix ${ }^{\circledR}$ Axiom ${ }^{\circledR}$ Maize Array (Unterseer et al. 2014).

After quality filtering and imputation, $910 \mathrm{DH}$ lines remained (501 lines in KE and 409 lines in PE) and the panel of markers reduced to 501,124 markers (Hölker et al. 2019). Additionally, loci which were in high level of pairwise linkage disequilibrium (LD) were removed (Calus and Vandenplas 2018) through linkage disequilibrium based SNP pruning with PLINK v1.07 (Purcell et al. 2007; Chang et al. 2015). LD pruning was done by the parameters of 50, 5 and 2 which considered as the SNPs window size, the number of SNPs at which the SNP window shifts and the variance inflation factor, respectively. This resulted in a data panel containing 25'437 SNPs for KE and 30 '212 SNPs for PE (Vojgani et al. 2020). Note that even a panel of $25^{\prime} 000$ SNPs results in more than 1 billion SNP interactions to account for. 
110 (TOM, Spain) were the four locations that these lines were phenotyped for a series of traits in 111 both 2017 and 2018.

112 The means, standard deviations, maximum and minimum values of studied phenotypic traits in 1132017 and 2018 in each landrace are compared in Table 1 which were derived from the Best Linear 114 Unbiased Estimations (BLUEs) of the genotype mean for each phenotypic trait by Hölker et al. 115 (2019). The comparison of the respective detailed values for each trait in each environment and 116 landrace in 2017 and 2018 are illustrated in the supplementary (Table S1). Vi in phenotypic traits 117 represents the vegetative growth stage when $i$ leaf collars are visible based on the leaf collar 118 method of the corn growth (Abendroth et al. 2011). Early vigour at V3 stage (EV_V3), female 119 flowering (FF) and root lodging (RL) were not phenotyped in all four environments for both years. 120 EV_V3 was not phenotyped in EIN in 2018, FF was not phenotyped in GOL in 2017 and RL was not 121 phenotyped in TOM and GOL in both 2017 and 2018.

122 The number of phenotyped lines per year and environment for trait PH_V4, as the main trait in 123 this study, are summarized in Table 2. For EIN and ROG a higher number of phenotyped lines were generated in 2017. On the contrary, more lines were phenotypes in GOL and TOM in 2018.

\section{Statistical models for phenotype prediction}

126

127

128

129

130

131

132

133

134

135

136

137

138

139

140

141

142

143

144

145

146

147

We used the bivariate statistical framework as the basis of the genomic prediction models. In this regard, GBLUP, ERRBLUP and SERRBLUP as three different methods described in Vojgani et al. (2020) were used for genomic prediction of phenotypes which differ in dispersion matrices representing their covariance structure of the genetic effects. GBLUP as an additive model is based on a genomic relationship matrix calculated according to VanRaden (2008). ERRBLUP (Epistatic Random Regression BLUP) as a full epistasis model is based on all pairwise SNP interactions which generates a new marker matrix considered as a marker combination matrix. The marker combination matrix is a 0,1 matrix indicating the absence (0) or presence (1) of each marker combination for each individual. sERRBLUP (selective Epistatic Random Regression BLUP) as a selective epistasis model is based on a selected subset of SNP interactions (Vojgani et al. 2019b). Vojgani et al. (2020) proposed estimated effect variances in the training set as the selection criterion of pairwise SNP interactions due to its robustness in predictive ability specifically when only a small proportion of interactions are maintained in the model.

\section{Assessment of genomic prediction models}

GBLUP, ERRBLUP and sERRBLUP models have been assessed via 5-fold cross validation by randomly partitioning the original sample into 5 equal size subsamples in which one subsample was considered as the test set to validate the model, and the remaining 4 subsamples were considered as a joint training set (Erbe et al. 2010). The 5-fold cross validation technique was utilized with 5 replicates through which the Pearson correlation between the predicted genetic values and the observed phenotypes in the test set was considered as the predictive ability in each fold of each replicate, which then was averaged across 25 replicates. In this study, predictive ability was separately assessed for KE and PE for a series of phenotypic traits in four different 
environments. Besides, we calculated the traits' prediction accuracies by dividing their predictive abilities by the square-root of the respective traits' heritabilities (Dekkers 2007) derived from all environments in both 2017 and 2018 jointly (Table S11 in the supplementary).

Univariate GBLUP within 2018 was assessed by training the model in the same year (2018) as the test set was sampled from. However, bivariate GBLUP, ERRBLUP and sERRBLUP were assessed by training the model with both the training set of the target environment in 2018 and the full dataset of the respective environment in 2017. The interaction selection step in bivariate sERRBLUP is done by first using the complete dataset of target environment in 2017 to estimate all pairwise SNP interaction effect variances. Then, an epistatic relationship matrix for all lines is constructed based on the subset of top ranked interaction effect variances, which is finally used to predict phenotypes of the target environment test set in 2018 (Vojgani et al. 2020).

\section{Variance component estimation}

Variance component estimation in univariate GBLUP was done by EMMREML (Akdemir and Godfrey 2015) based on the training set in each run of 5-fold cross validation with 5 replicates. In bivariate models this was done by ASReml-R (Butler et al. 2018) with the approach specified by Vojgani et al. (2020) for pre estimating the variance components from the full dataset to derive the initial values for the variance components in ASReml models in 100 iterations for each combination. If the variance estimation based on the full set did not converge after 100 iterations, then the estimated variance components at the $100^{\text {th }}$ iteration were extracted as initial values of the bivariate model in the cross validation step. Afterwards, the model used these values to re-estimate the variance components based on the training set in each run of 5-fold cross validation in 50 iterations. The estimated variance components in the converged models based on the full set deviated only slightly from the estimated variance components based on the training set (Fig. 1). However, the variance component estimations did not converge in all folds of 5 -fold cross validation with 5 replicates. In such cases, the initial values were set as the fixed values for the model to predict the breeding values. This approach appears justifiable in the case of non-convergence of the bivariate model, since we have shown in Fig. 2 that the difference in mean predictive ability of all folds and only the converged folds is not critical. This difference can get higher as the number of non-converged folds increases. The number of not converged folds in all studied material is shown in the supplementary (Table S12).

\section{Genomic correlation estimation}

Genomic correlations were estimated from the genetic variances and covariance derived from the ASReml bivariate model based on the full dataset of each environment in both 2017 and 2018. 
Table 1: Phenotypic trait description and the mean, minimum, maximum and standard deviation of the BLUEs for each phenotypic trait in KE and PE landraces in the years 2017 and 2018.

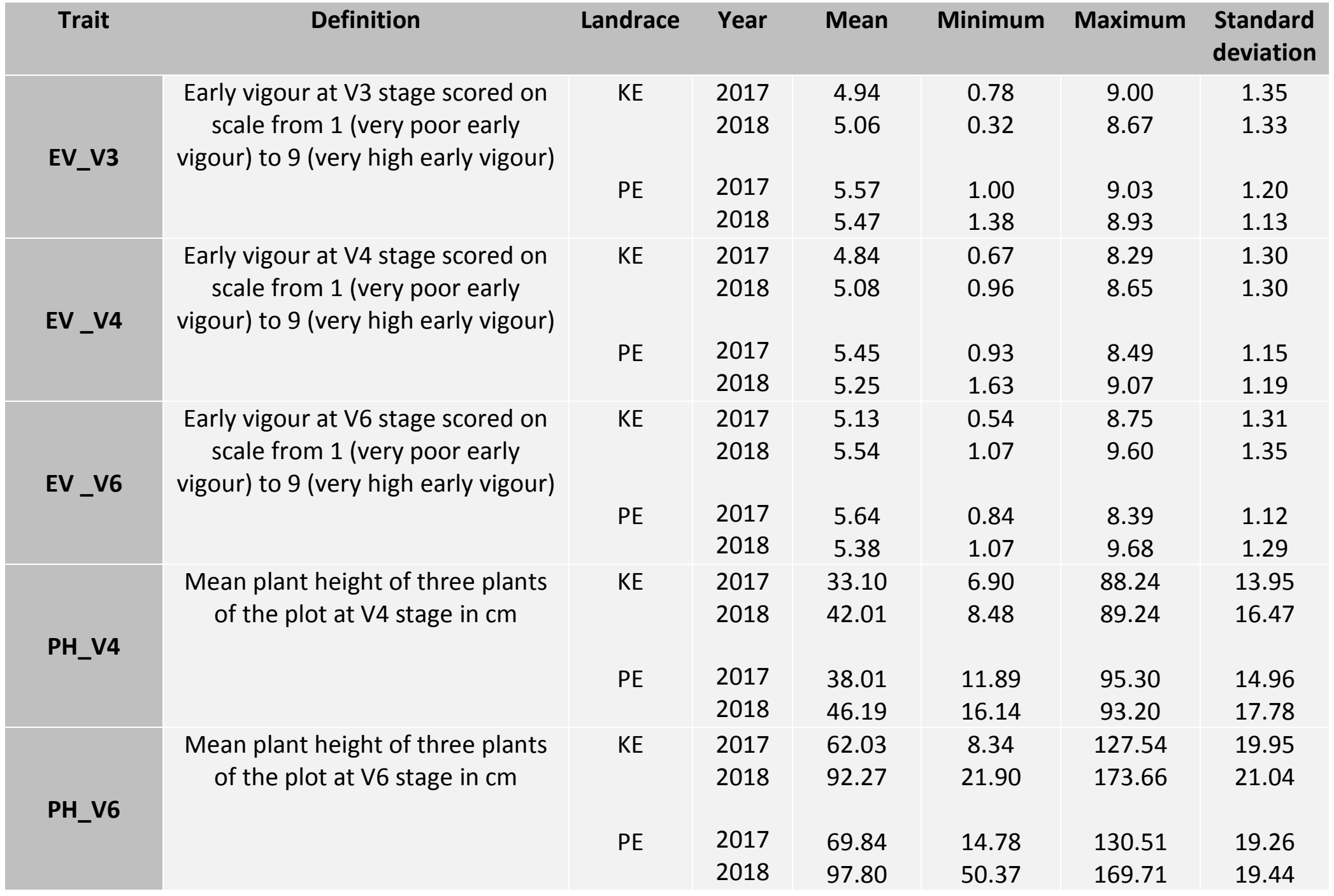




\begin{tabular}{|c|c|c|c|c|c|c|c|}
\hline \multirow[b]{2}{*}{ PH_final } & $\begin{array}{l}\text { Final plant height after flowering in } \\
\qquad \mathrm{cm}\end{array}$ & KE & $\begin{array}{l}2017 \\
2018\end{array}$ & $\begin{array}{l}139.10 \\
146.04\end{array}$ & $\begin{array}{l}49.27 \\
35.41\end{array}$ & $\begin{array}{l}245.00 \\
265.02\end{array}$ & $\begin{array}{l}27.14 \\
35.74\end{array}$ \\
\hline & & PE & $\begin{array}{l}2017 \\
2018\end{array}$ & $\begin{array}{l}124.09 \\
128.08\end{array}$ & $\begin{array}{l}30.21 \\
35.76\end{array}$ & $\begin{array}{l}211.14 \\
248.43\end{array}$ & $\begin{array}{l}24.54 \\
35.99\end{array}$ \\
\hline \multirow[t]{2}{*}{ FF } & $\begin{array}{l}\text { Days after sowing until female } \\
\text { flowering (days until } 50 \% \text { of the } \\
\text { plot showed silks) }\end{array}$ & KE & $\begin{array}{l}2017 \\
2018\end{array}$ & $\begin{array}{l}79.72 \\
76.99\end{array}$ & $\begin{array}{l}62.45 \\
62.22\end{array}$ & $\begin{array}{l}102.02 \\
100.14\end{array}$ & $\begin{array}{l}6.27 \\
6.09\end{array}$ \\
\hline & & PE & $\begin{array}{l}2017 \\
2018\end{array}$ & $\begin{array}{l}78.85 \\
76.70\end{array}$ & $\begin{array}{l}59.10 \\
60.14\end{array}$ & $\begin{array}{c}101.50 \\
93.96\end{array}$ & $\begin{array}{l}6.33 \\
6.52\end{array}$ \\
\hline \multirow{2}{*}{$\mathbf{R L}$} & $\begin{array}{l}\text { Root lodging score from } 1 \text { to } 9 \text { ( } 1= \\
\text { no lodging and } 9=\text { severe lodging) }\end{array}$ & KE & $\begin{array}{l}2017 \\
2018\end{array}$ & $\begin{array}{l}3.38 \\
1.42\end{array}$ & $\begin{array}{l}0.59 \\
0.73\end{array}$ & $\begin{array}{l}9.58 \\
8.52\end{array}$ & $\begin{array}{l}2.50 \\
0.90\end{array}$ \\
\hline & & PE & $\begin{array}{l}2017 \\
2018\end{array}$ & $\begin{array}{l}2.14 \\
1.21\end{array}$ & $\begin{array}{l}0.03 \\
0.32\end{array}$ & $\begin{array}{l}9.22 \\
4.69\end{array}$ & $\begin{array}{l}1.74 \\
0.51\end{array}$ \\
\hline
\end{tabular}


Table 2: Number of KE and PE lines phenotyped in each location for the years 2017 (blue numbers) and 2018 (red numbers) for trait PH_V4.

\begin{tabular}{|lcccc|}
\hline & EIN & ROG & GOL & TOM \\
\hline & $(2017 \backslash 2018)$ & $(2017 \backslash 2018)$ & (2017\2018) & (2017 2018$)$ \\
\hline Phenotyped lines in KE & $462 \backslash 365$ & $461 \backslash 365$ & $211 \backslash 222$ & $211 \backslash 222$ \\
\hline Phenotyped lines in PE & $393 \backslash 365$ & $390 \backslash 365$ & $204 \backslash 240$ & $204 \backslash 240$ \\
\hline
\end{tabular}

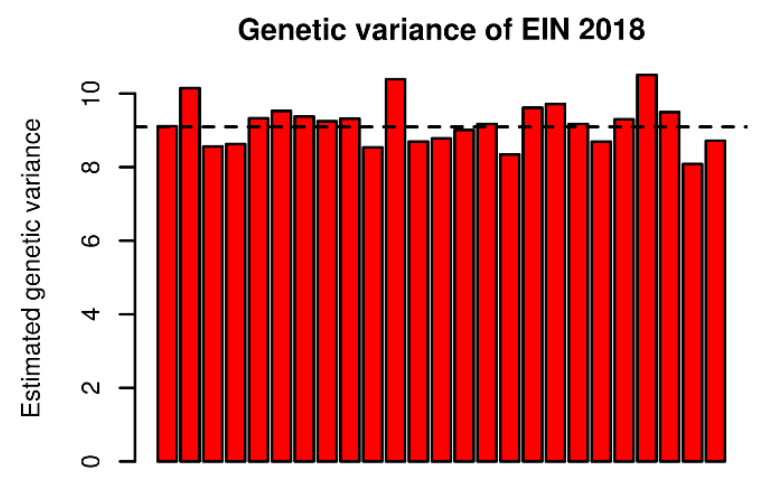

Residual variance of EIN 2018

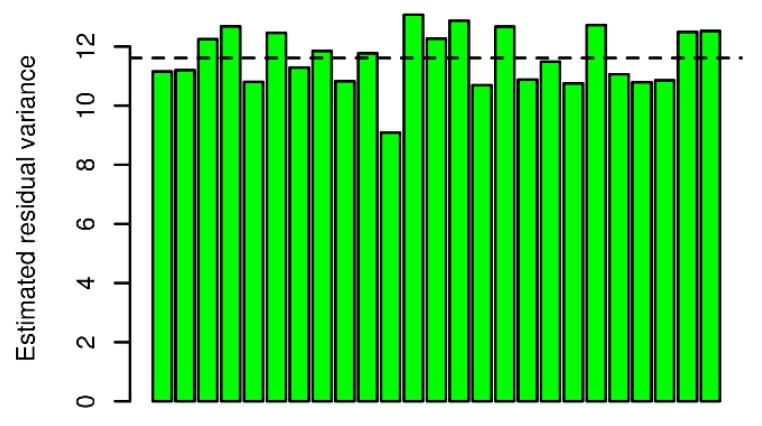

Genetic covariance of EIN 2018 and EIN 2017

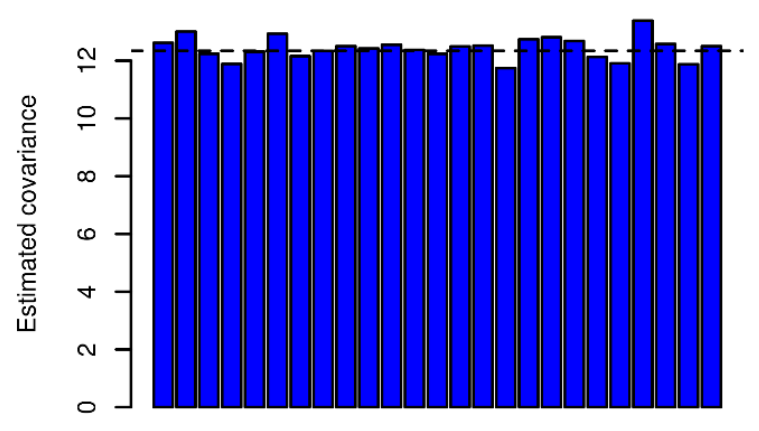

Genetic variance of EIN 2017

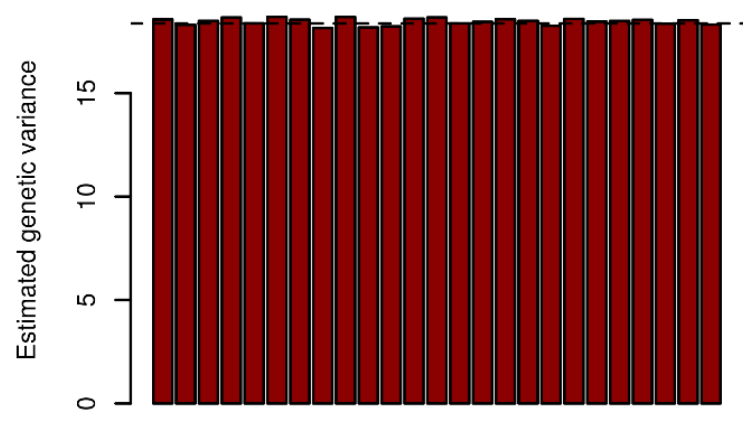

Residual variance of EIN 2017

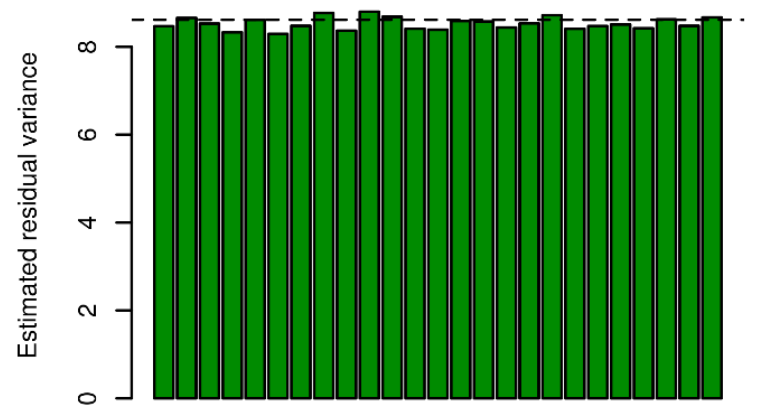

Residual covariance of EIN 2018 and EIN 2017

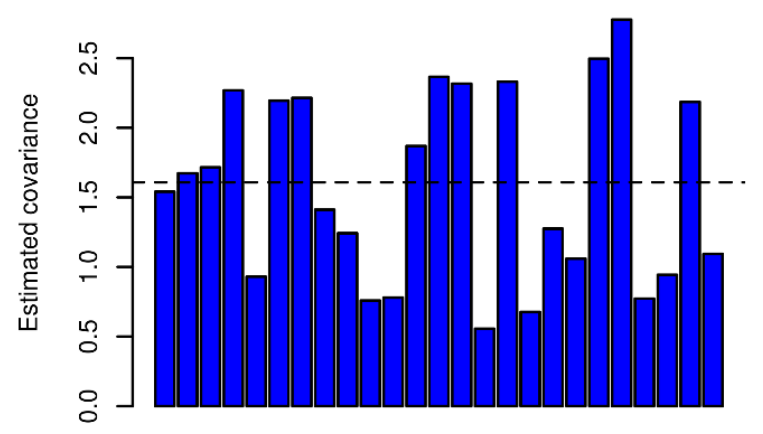

Fig. 1: Comparison of pre estimated genetic and residual variances and covariances of converged bivariate sERRBLUP (top 10\%) based on the full dataset (dashed horizontal lines) and estimated genetic and residual variances and covariances of converged bivariate SERRBLUP (top 10\%) based on training set in each run of 5-fold cross validation with 5 replicates (colored bars) for predicting EIN in 2018 when the additional environment is EIN in 2017 in KE for trait PH-V4. 
193

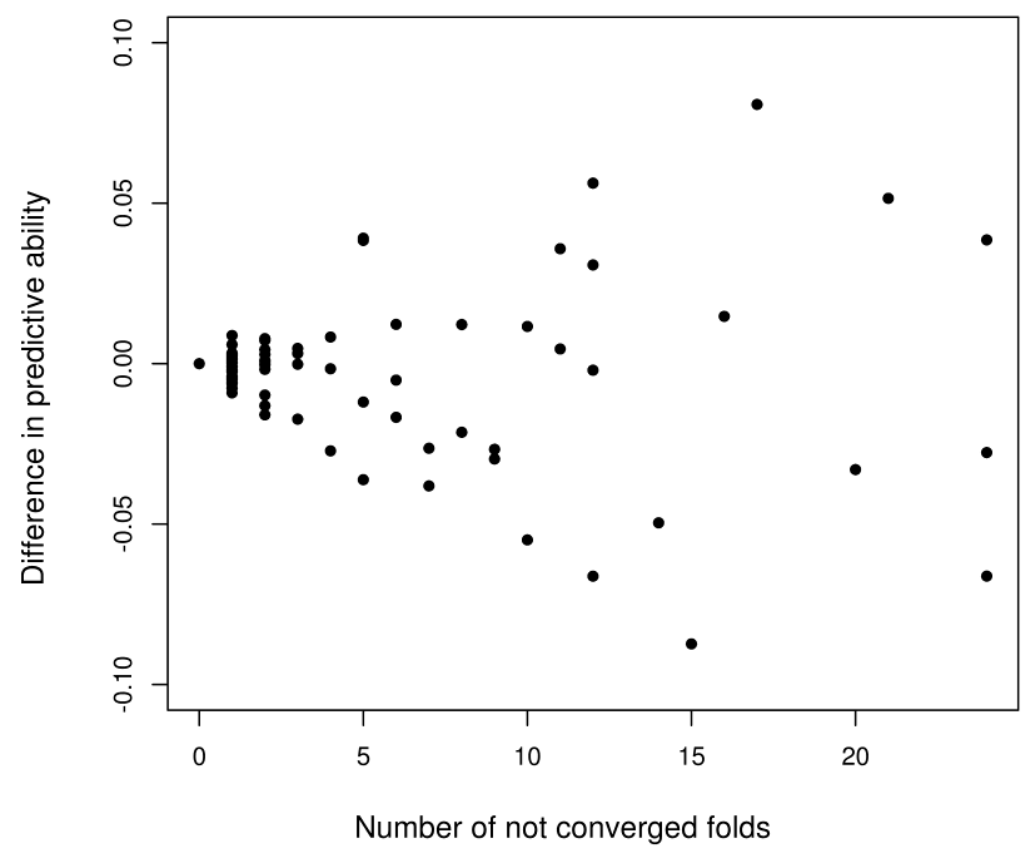

Fig. 2: The difference between the mean predictive ability of only the converged folds and the mean predictive ability of all folds in 5-fold cross validation with 5 replicates virus the number of the folds which did not converged across all traits in all combinations for both KE and PE in bivariate GBLUP, ERRBLUP, SERRBLUP.

\section{Results}

Bivariate models outperform the univariate models (Vojgani et al. 2020) and this has been confirmed in our study through the comparison in predictive ability of bivariate GBLUP and univariate GBLUP for the trait PH-V4 in both landraces indicating the superiority of bivariate GBLUP to univariate GBLUP in most cases (see Fig. 3). Among the bivariate genomic prediction models, bivariate ERRBLUP increases the predictive ability only slightly compared to bivariate GBLUP in a range from +0.008 to +0.024 for the trait PH-V4 across all environments in both landraces. This predictive ability increases further in bivariate SERRBLUP and the highest gain in accuracy is generally obtained when the top 10 or 5 percent of pairwise SNP interactions kept in the model in most cases. A too strict selection like using only the top 0.001 percent interactions, results in a decrease in predictive ability (see Fig. 3). Robustness of the predictive ability depending on the share of selected markers was higher in PE. Similar patterns are observed across a series of other traits for bivariate models which are shown in the supplementary (Fig. S1-S7). Additionally, the predictive ability of univariate GBLUP by training the model on the average phenotypic values of both 2017 and 2018 was evaluated for a series of phenotypic traits, which yielded quite similar predictive ability as obtained with univariate GBLUP within year 2018 or worse in some cases (Table S10a (KE) and S10b (PE) in supplementary). 
Einbeck

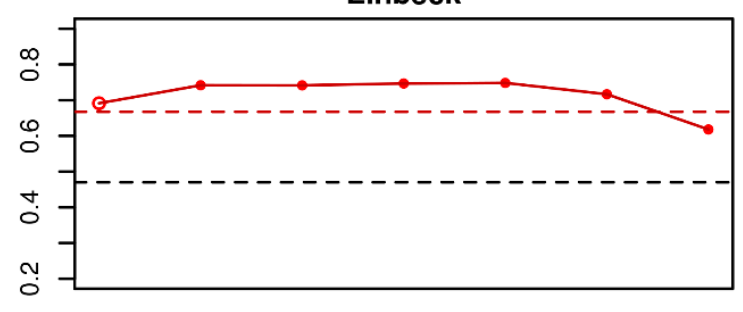

Roggenstein

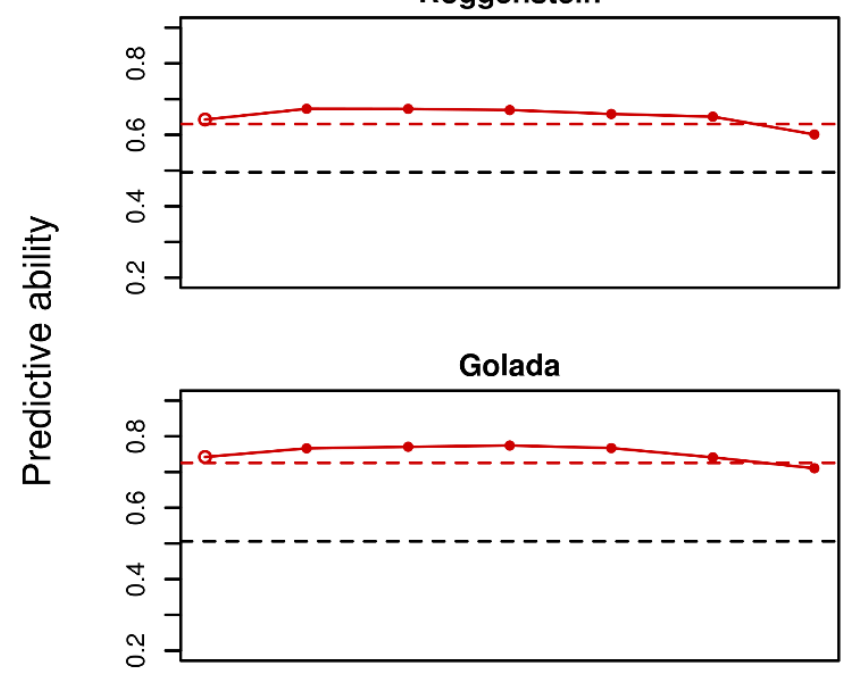

Tomeza

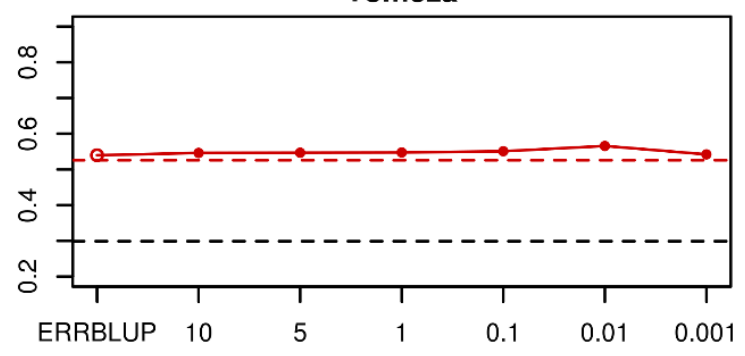

Einbeck

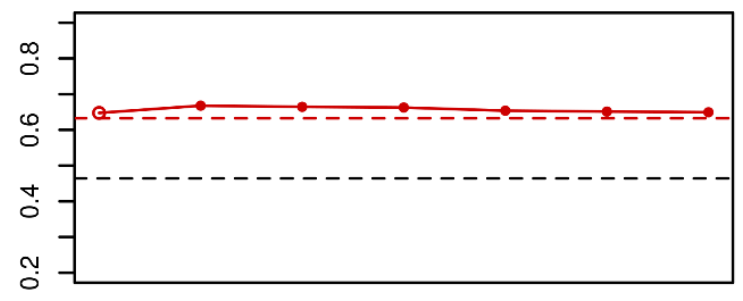

Roggenstein

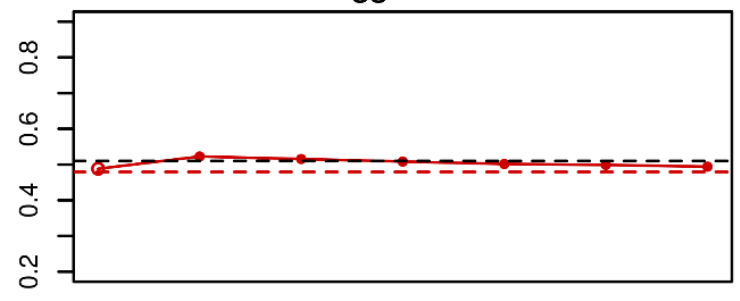

Golada

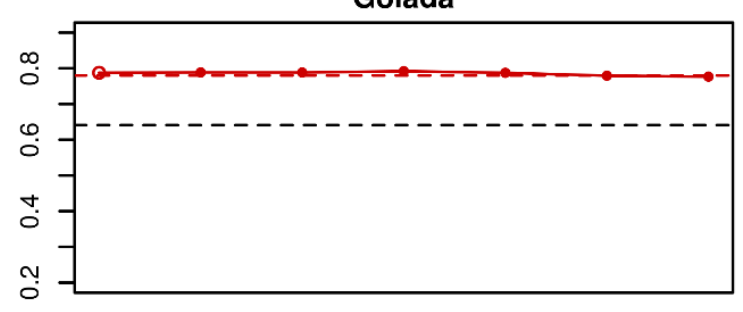

Tomeza

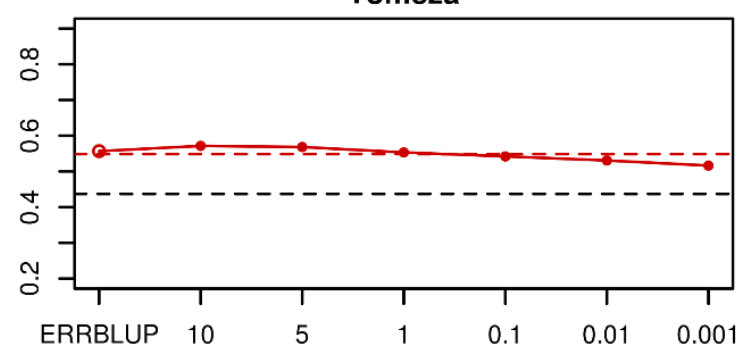

Percentage of interactions maintained in the model

Fig. 3: Predictive ability for univariate GBLUP within 2018 (black dashed horizontal line), bivariate GBLUP (red dashed horizontal line), bivariate ERRBLUP (red open circle) and bivariate sERRBLUP (red filled circles and red solid line) for trait PH-V4 in KE (left) and in PE (right).

The absolute gain in predictive ability from univariate GBLUP to maximum bivariate sERRBLUP was regressed on the respective SERRBLUP genomic correlation between the two respective environment and across the series of studied traits (Fig. 4). Regression coefficients range between 0.09 and 0.51 and thus show a clear association between the absolute gain in prediction accuracy and the genomic correlation between environments. When combining all traits and 
environments, this correlation is $0.64(p$-value $=0.00024)$ in $\mathrm{KE}$ and $0.73(p$-value $=1.072 \mathrm{e}-05)$ in

PE.
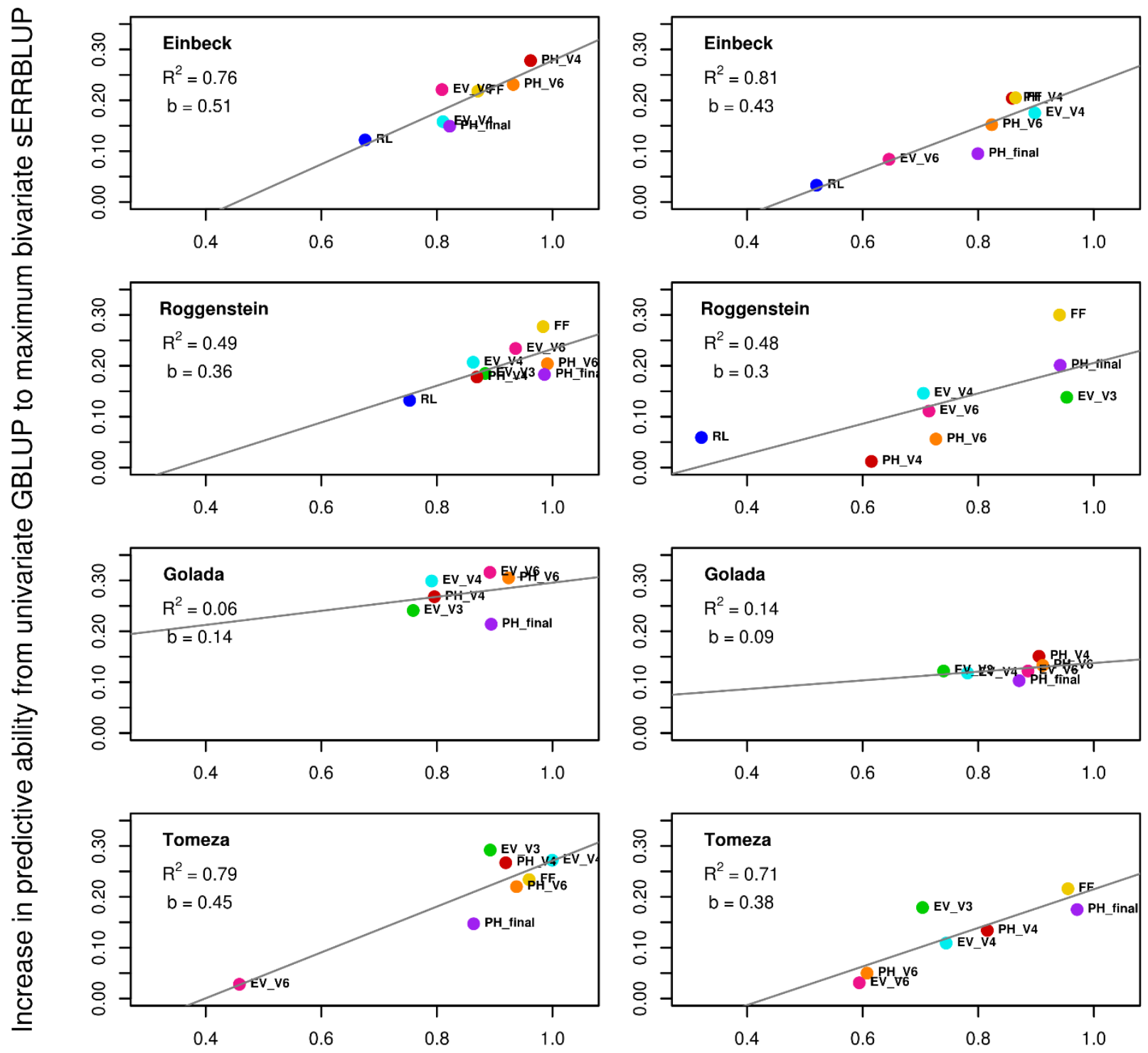

sERRBLUP genomic correlation

227 Fig. 4: Regression of the absolute increase in predictive ability from univariate GBLUP to maximum 228 bivariate sERRBLUP on the respective sERRBLUP genomic correlation between 2017 and 2018 in KE (left) 229 and in PE (right) for all studied traits. In each panel, the overall linear regression line (gray solid line) with 230 the regression coefficient $(\boldsymbol{b})$ and R-squared $\left(\boldsymbol{R}^{2}\right)$ are shown.

231 The genomic correlations across years estimated with GBLUP and sERRBLUP for the trait PH_V4 232 are illustrated in Table 3, indicating that the proportion of interactions in bivariate SERRBLUP 233 which maximized the predictive ability are not necessarily linked to the highest genomic 
correlation. In contrast, the best sERRBLUP for trait PH_V4 is linked to the lowest genomic correlation in most cases. However, this is not the general pattern observed for series of other traits and the best SERRBLUP for some traits and environments combinations are linked to the highest genomic correlation (Table S3-S9 in supplementary).

Table 3: Genomic correlation between 2017 and 2018 in each environment for trait PH_V4 for KE (blue numbers) and PE (red numbers). The blue and red bold numbers with stars indicate which proportion of interactions in bivariate SERRBLUP maximized the predictive ability in each environment for KE and PE, respectively.

\begin{tabular}{|c|c|c|c|c|}
\hline Bivariate Models & EIN & ROG & GOL & TOM \\
\hline GBLUP & 0.945 / 0.898 & 0.940 / 0.658 & 0.942 / 0.969 & 0.954 / 0.923 \\
\hline sERRBLUP top $10 \%$ & 0.955 / 0.859* & $0.869 * / 0.615 *$ & 0.835 / 0.895 & $0.929 / 0.816^{*}$ \\
\hline sERRBLUP top 5\% & $0.958 / 0.868$ & 0.850 / 0.631 & 0.797 / 0.888 & 0.912 / 0.826 \\
\hline sERRBLUP top $1 \%$ & $0.949 * / 0.895$ & $0.848 / 0.820$ & $0.796 * / 0.905 *$ & $0.918 / 0.863$ \\
\hline sERRBLUP top $0.1 \%$ & 0.962 / 0.966 & 0.917 / 0.922 & 0.884 / 0.948 & 0.929 / 0.959 \\
\hline sERRBLUP top $0.01 \%$ & $0.963 / 0.980$ & $0.951 / 0.985$ & $0.911 / 0.983$ & $0.919 * / 0.987$ \\
\hline sERRBLUP top $0.001 \%$ & $0.997 / 0.976$ & $0.963 / 0.970$ & $0.908 / 0.973$ & $0.933 / 0.968$ \\
\hline
\end{tabular}

In this regard, the absolute increase in predictive ability from bivariate GBLUP to maximum bivariate SERRBLUP was regressed on the difference between genetic correlations estimated with GBLUP and maximum SERRBLUP, respectively, across all traits in both landraces. Fig. 5 shows a significant correlation of 0.42 ( $p$-value $=0.0255)$ in $K E$ and $0.74(p$-value $=6.458 \mathrm{e}-06)$ in PE between the absolute gain in the respective predictive ability and the difference in the corresponding genetic correlations. 

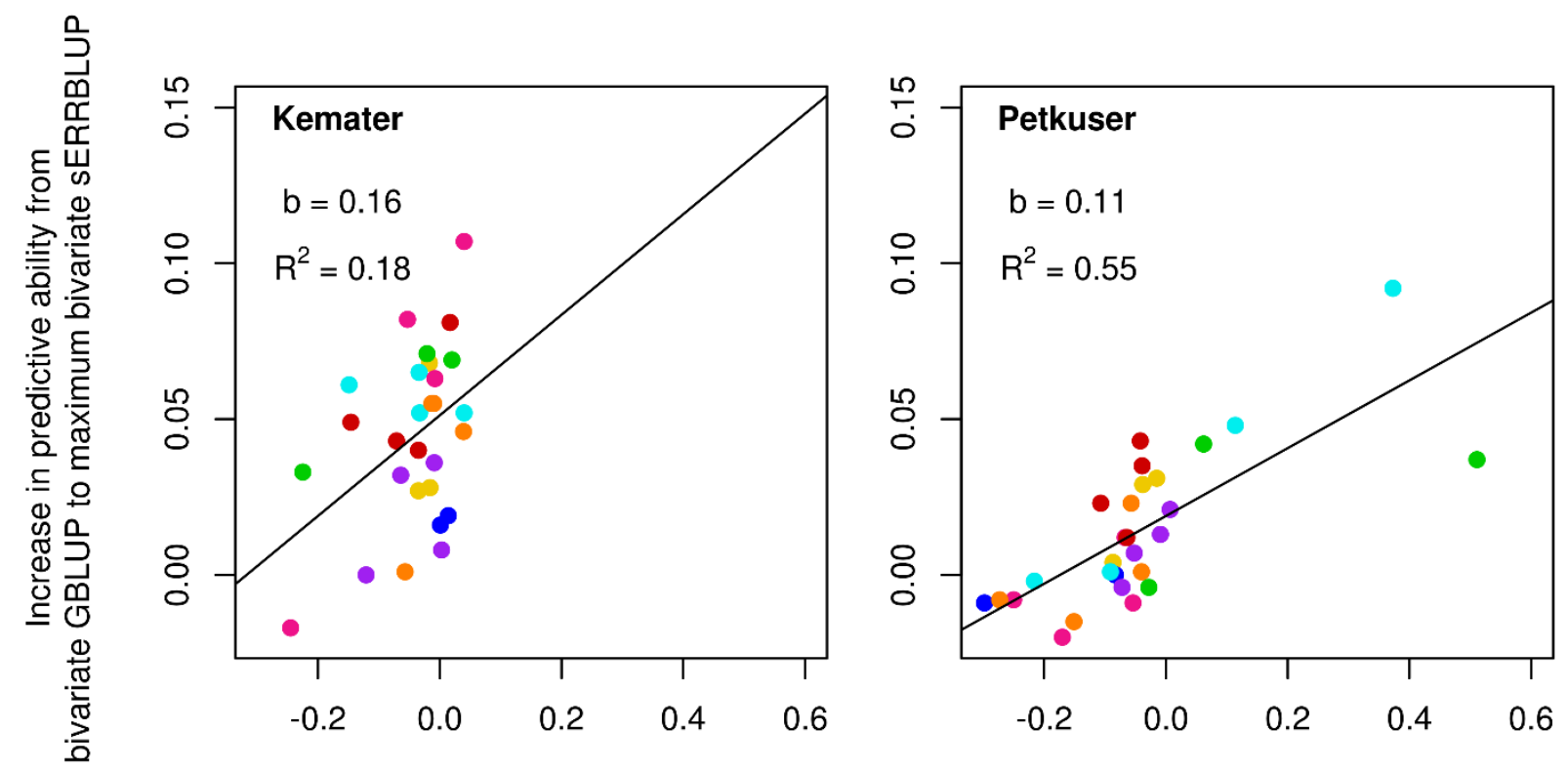

Difference in GBLUP and maximum sERBLUP Genomic correlation

Fig. 5: Regression of the absolute increase in predictive ability from bivariate GBLUP to maximum bivariate SERRBLUP on the difference between the GBLUP genomic correlation and maximum sERRBLUP genomic correlation between 2017 and 2018 in KE (left) and in PE (right) for all studied traits. In each panel, the overall linear regression line with the regression coefficient $(\boldsymbol{b})$ and $\mathrm{R}$-squared $\left(\boldsymbol{R}^{2}\right)$ are shown. The colors green, light blue, pink, red, orange, purple, yellow and dark blue represent the phenotypic traits EV_V3, EV_V4, EV_V6, PH_V4, PH_V6, PH_final, FF and RL, respectively.

There might be some tendency that including phenotypes of the previous year into prediction becomes more efficient when the phenotypic correlation between years is high. In this context, the correlation between the absolute gain in predictive ability from univariate GBLUP to maximum bivariate sERRBLUP and the phenotypic correlation among the years (see Table S2) over all studied traits in all four environments and in both landraces was studied. Fig. 6 demonstrates that the maximum correlation between the absolute gain in the respective predictive ability and the phenotypic correlation is obtained in EIN for KE (0.69) and in TOM for $P E(0.72)$. Across all studied traits and environments, there is a significant correlation of 0.59 in $\mathrm{KE}(p$-value $=0.001)$ and 0.47 in PE (p-value $=0.01)$. 

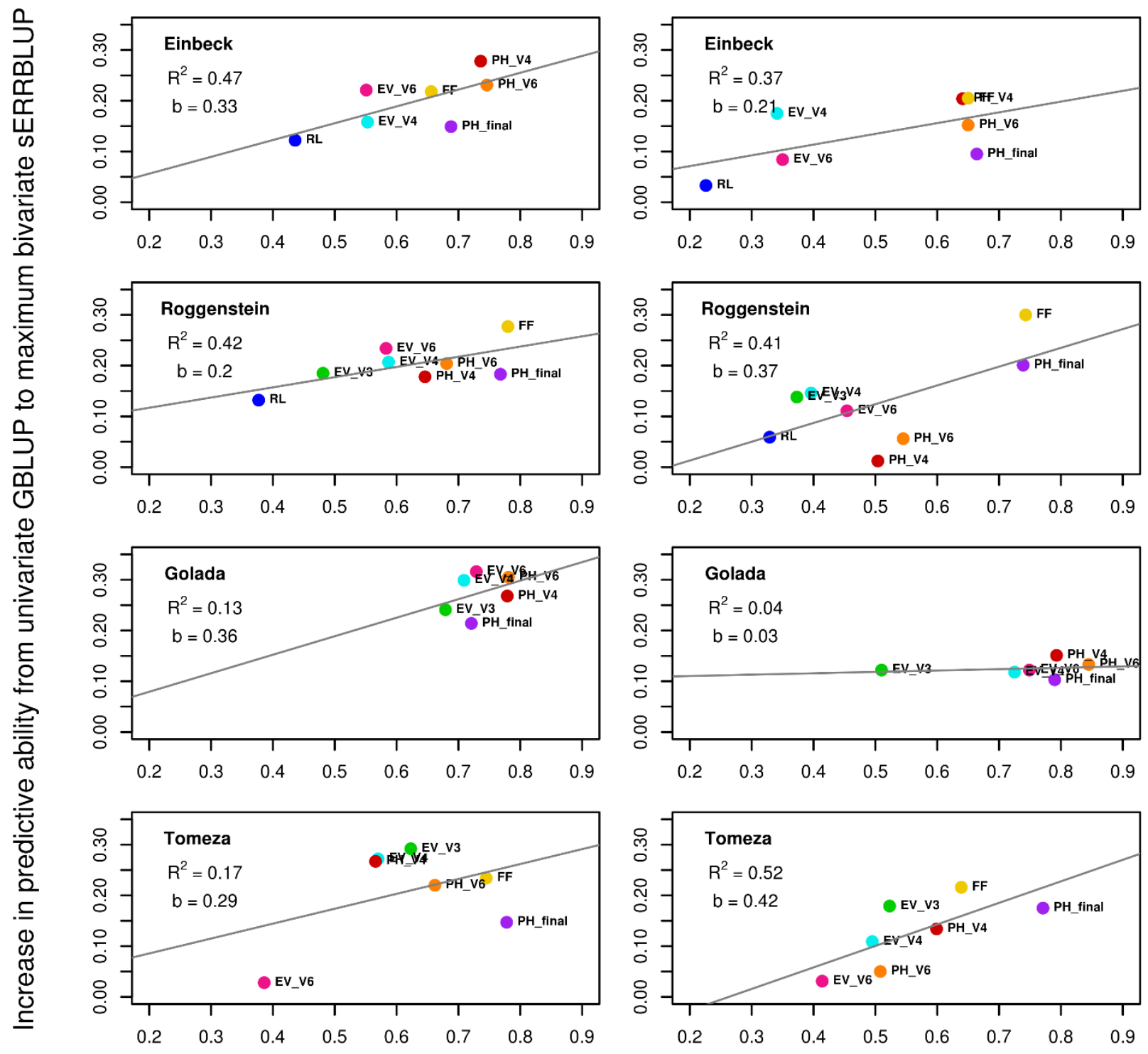

Phenotypic correlation

267 Fig. 6: Regression of the absolute increase in predictive ability from univariate GBLUP to maximum 268 bivariate sERRBLUP on the phenotypic correlation between 2017 and 2018 in KE (left) and in PE (right) for 269 all studied traits. In each panel, the overall linear regression line (gray solid line) with the regression 270 coefficient $(\boldsymbol{b})$ and R-squared $\left(\boldsymbol{R}^{2}\right)$ are shown.

271 Overall, the percentage of relative increase in prediction accuracy from the bivariate GBLUP to 272 the maximum bivariate SERRBLUP in both landraces reveals more increase in prediction accuracy 273 for KE than PE with the average increase of 7.61 percent in KE and 3.47 percent in PE over all 274 studied traits (see Fig. 7). Among all traits, the maximum increase in prediction accuracy for KE is 27522.63 percent which was obtained in EV_V6 in EIN, and for PE is 34.59 percent which was 
obtained in EV_V4 in EIN. However, Fig. 7 shows some slight decreases in prediction accuracy from bivariate GBLUP to maximum bivariate sERRBLUP for some combinations of traits and environment in both landraces. This is more often observed in PE than KE, where the maximum decrease was found in EV_V6 in TOM for both PE (-3.198 percent) and KE (-2.795 percent). Overall, the average relative increase from bivariate GBLUP to maximum bivariate sERRBLUP was over 3 percent in most cases. The absolute increase in prediction accuracy is also illustrated in the supplementary (Fig. S8) indicating the average increase of 0.046 in KE and 0.015 in PE over all combinations of traits and environments.
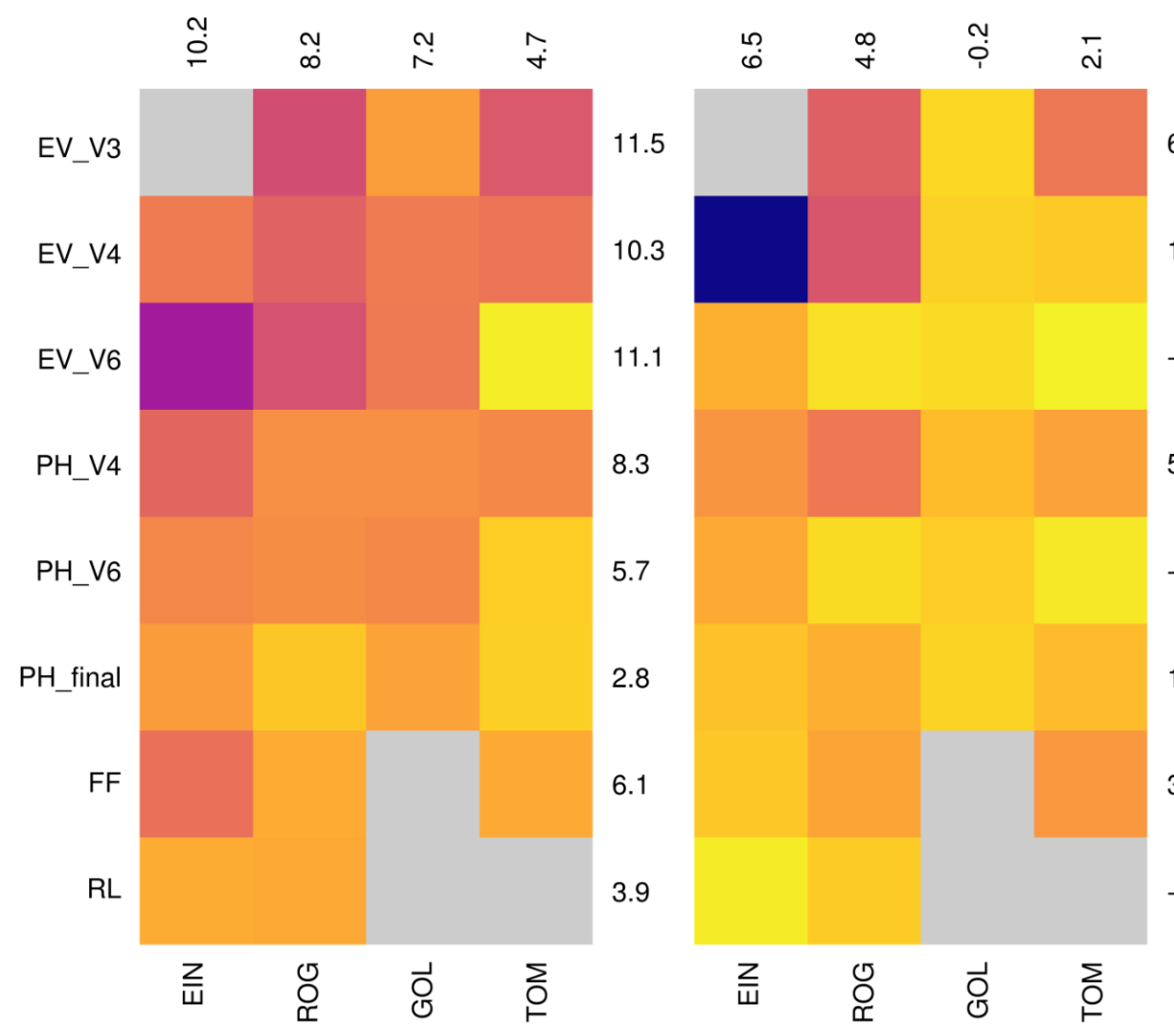

6.7 Percentage of increase in

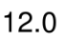
prediction accuracy

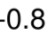

5.1

$-0.1$

1.3

$-1.3$

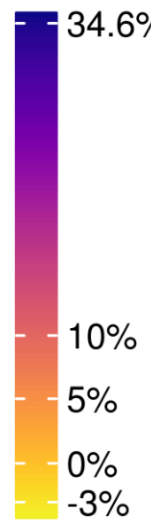

No data

Fig. 7: Percentage of change in prediction accuracy from bivariate GBLUP to the maximum prediction accuracy of bivariate sERRBLUP in KE (left side plot) and in PE (right side plot). The average percentage of change in prediction accuracy for each trait and environment is displayed in all rows and columns, respectively.

Finally, a comparison between the absolute increase in prediction accuracy from bivariate GBLUP to maximum bivariate SERRBLUP in PE versus KE shows a higher increase in KE compared to PE with a regression coefficient 0.25 (see Fig. 8). This indicates some consistency of the observed trends across landraces. This was also confirmed with paired t-test indicating that the mean increase in prediction accuracy for KE is significantly higher than in PE ( $p$-value $=3.921 e-05)$. 


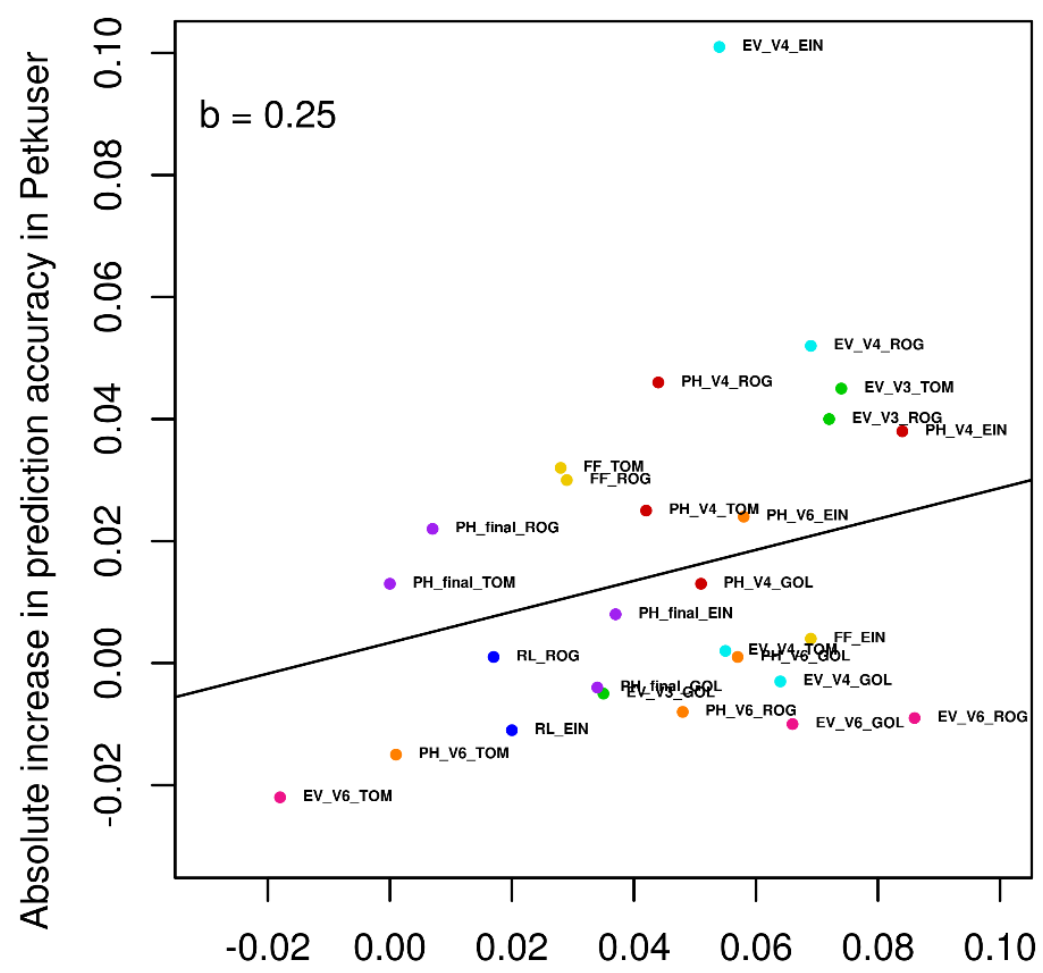

Absolute increase in prediction accuracy in Kemater

Fig. 8: Absolute change in prediction accuracy from bivariate GBLUP to the maximum prediction accuracy of bivariate sERRBLUP in PE vs. KE. The black line represents the overall linear regression line.

\section{Discussion}

In this study, bivariate ERRBLUP as a full epistasis model incorporating all pairwise SNP interactions provides only a modest increase in predictive ability compared to bivariate GBLUP. This was expected, since ERRBLUP incorporates a high number of interactions by which a large number of unimportant variables are introduced into the model (Martini et al. 2016), thus introducing potential 'noise' which can prevent gains in predictive ability. In contrast, bivariate sERRBLUP substantially increases the predictive ability compared to bivariate GBLUP. In fact, the increase in predictive ability from bivariate GBLUP to bivariate sERRBLUP is only caused by inclusion of relevant pairwise SNP interactions. Note that all bivariate models substantially outperformed univariate GBLUP, as phenotypic data of the respective environment in the previous year was used.

It was shown that multivariate GBLUP is superior in predictive ability compared to univariate GBLUP under existence of medium $(\sim 0.6)$ or high ( $\sim 0.9)$ genomic correlation, and that the low genomic correlation results in no increase in multivariate GBLUP compared to univariate GBLUP (Covarrubias-Pazaran et al. 2018). Calus et al. (2011) also found an increase of 3 to 14 percent in 312 predictive ability of multi-trait SNP-based models in a simulation study when genetic correlations 
ranged from 0.25 to 0.75 . In our study, we also found a significant correlation between the absolute gain in prediction accuracy from univariate GBLUP to maximum bivariate sERRBLUP and the respective genomic correlation in both KE $(r=0.64)$ and PE $(r=0.73)$ across all traits and environments combinations.

Moreover, Martini et al. (2016) showed that the predictive ability in one environment can be increased by variable selection in the other environment under the assumption of positive phenotypic correlation between environments. It was shown in a wheat dataset (Pérez and de los Campos 2014), where environments 2 and 3 had the highest phenotypic correlation (0.661), that the predictive ability for phenotype prediction in environment 2 was maximized by variable selection in environment 3 and vice versa (Martini et al. 2016). Therefore, the increase in prediction accuracy is expected to be influenced by the phenotypic correlations between the environments or between the years in the same environment in bivariate models. In our study, although 2017 and 2018 were climatically quite different, since 2018 suffered from a major heat stress compared to 2017 (Table 1), we see a significant correlation between the absolute gain in predictive ability from univariate GBLUP to maximum predictive ability of bivariate sERRBLUP and the phenotypic correlation between years in each environment for both KE $(r=0.59)$ and PE $(r=0.47)$.

In addition to the genomic and phenotypic correlations between the years, the trait heritability is another factor which is expected to be influential for such an increase in bivariate sERRBLUP predictive ability as well. Therefore, the traits with lower heritability are expected to obtain less gain in SERRBLUP predictive ability than the traits with higher heritability. In our study, the correlation between the absolute gain in prediction accuracy from univariate GBLUP to maximum bivariate SERRBLUP and a trait's heritability over all studied material was considerable in both KE $(r=0.35)$ and PE ( $r=0.45)$ (Fig. S9 in the supplementary). Based on the obtained results, the traits with low heritability (e.g. 0.59 for RL in PE) showed only a small increase in prediction accuracy. However, not all traits with higher heritabilities did necessarily show a higher gain in predictive ability for all traits. Overall, this association between the absolute gain in predictive ability and the trait heritabilities were close to significant in KE ( $p$-value $=0.07$ ) and highly significant in PE ( $p$-value=0.02). It should be noted that the trait heritabilities were calculated on an entry-mean basis within each KE and PE landraces (Hallauer et al. 2010) over all eight environments in both years 2017 and 2018 jointly. The trait heritabilities obtained only from 2017 are significantly higher than the trait heritabilities obtained only from 2018 in both KE and PE based on a paired t-test (Table S11 in the supplementary). This also results in an increase in predictive ability from univariate GBLUP to maximum bivariate sERRBLUP in KE and PE, since multi-trait models have the potential of increasing the predictive ability when traits with low heritability are joined with traits with higher heritability, given they are genomically correlated (Thompson and Meyer 1986). 
It should be noted that the increase in predictive ability from univariate GBLUP to maximum bivariate SERRBLUP is caused by both borrowing information across years and capitalizing on epistasis, while the increase in predictive ability from bivariate GBLUP to maximum bivariate SERRBLUP is caused by accounting for epistasis alone. Overall, the traits behave differently among different environments and landraces due to their genomic correlations, phenotypic correlations and heritabilities. To shed light on this, the maximum increase in prediction accuracy from bivariate GBLUP to bivariate sERRBLUP in KE was observed for the trait EV_V6 (0.112) in EIN where the corresponding SERRBLUP genomic correlation (0.809) is higher than the GBLUP genomic correlation (0.768). This trait has a high heritability (0.90) and high phenotypic correlation (0.551) as well. In contrast, the respective prediction accuracy decreases (-0.018) for EV_V6 in TOM for KE indicating the lower sERRBLUP genomic correlation (0.458) than GBLUP genomic correlation (0.703) and the particularly low phenotypic correlation (0.383). It should be noted that the phenotypic correlation does not play a major role for the increase in prediction accuracy from bivariate GBLUP to bivariate SERRBLUP, since both models are bivariate and benefit from the same phenotypic correlations. Therefore, EV_V6 obtaining the maximum and minimum increase in the respective prediction accuracy for KE indicates the significant role of genomic correlation among the possible causes. In general, bivariate sERRBLUP improves the prediction accuracy compared to bivariate GBLUP more in KE than PE which is potentially due to significantly higher sERRBLUP genomic correlation and heritability in KE compared to PE, based on paired t-test.

In our study, 5-fold cross validation with 5 replicates was utilized to evaluate our bivariate genomic prediction models. Different split of cross validation such as 10 -fold cross validation did not make a considerable difference in our bivariate models' predictive abilities (Fig. S10 in the supplementary). The maximum increase in bivariate models' predictive abilities when utilizing 10-fold cross validation with 10 replicates compared to utilizing 5 -fold cross validation with 5 replicates was 0.018 in KE and 0.006 in PE for trait PH_V4. Overall, our cross validation scenario is not expected to bias the predictive abilities obtained from our bivariate models for reasons as outlined by Runcie and Cheng (2019), who observed a bias when the test set of the target trait is predicted from the full dataset of the second trait in multi-trait model. In our study, utilizing the full dataset of the target trait in one environment from 2017 to predict the same biological trait in the respective environment in 2018 should not lead to such a bias in predictive ability, since the individuals do not share the same source of non-genetic variation and they have been grown in two different years which have been climatically very different from each other.

Overall, our results indicate that incorporating a suitable subset of epistatic interactions besides utilizing information across years can substantially increase the predictive ability. The amount of this increase is affected by the genomic and phenotypic correlations between the years and the heritability of the phenotypic trait. Therefore, this approach is potentially beneficial for genomic prediction of phenotypes under the assumption of sufficient genomic and phenotypic correlation between years for highly heritable traits. This may allow to reduce the number of lines which 
bioRxiv preprint doi: https://doi.org/10.1101/2020.11.18.388330; this version posted November 20, 2020. The copyright holder for this preprint (which was not certified by peer review) is the author/funder. All rights reserved. No reuse allowed without permission.

389 have to be phenotyped over several years and thus reduce phenotyping costs which and thus be 390 of high interest in practical plant breeding.

391 


\section{Declaration}

\section{Funding}

394 This work was funded by German Federal Ministry of Education and Research (BMBF) within the 395 scope of the funding initiative "Plant Breeding Research for the Bioeconomy" (MAZE - "Accessing 396 the genomic and functional diversity of maize to improve quantitative traits"; Funding ID: 397 031B0195)

398 Conflict of interest

399 On behalf of all authors, the corresponding author states that there is no conflict of interest.

$400 \quad$ Ethics approval

401 The authors declare that this study complies with the current laws of the countries in which the 402 experiments were performed.

403 Consent to participate

404 Not applicable

405 Consent for publication

406 Not applicable

407 Availability of data and materials

408 All data and material are available through material transfer agreements upon request.

409 Code availability

$410 \quad$ Not applicable

\section{Authors' contributions}

412 EV derived the results, analyzed the data, wrote the manuscript; TP proposed epistasis 413 relationship matrices; $\mathrm{ACH}, \mathrm{MM}$ and CCS prepared the material; $\mathrm{ACH}$ proposed cross validation 414 strategy in bivariate model; HS proposed the original research question, guided the structure of 415 the research.TP ACM MM CCS HS read, revised and approved the manuscript.

416 Corresponding author

417 Correspondence to Elaheh Vojgani

418 Email: vojgani@gwdg.de

419 ORCID: 0000-0003-4375-3531 


\section{Acknowledgements}

422 We are thankful to KWS SAAT SE, Misión Biológica de Galicia, Spanish National Research Council 423 (CSIC), Technical University of Munich, and University of Hohenheim for providing the extensive 424 phenotypic evaluation. We are grateful to the German Federal Ministry of Education and 425 Research (BMBF) for the funding of our project within the scope of the funding initiative "Plant 426 Breeding Research for the Bioeconomy" (MAZE - "Accessing the genomic and functional diversity 427 of maize to improve quantitative traits"; Funding ID: 031B0195). 


\section{References}

Abendroth L, Elmore RW, Boyer MJ, and Marlay SK (2011) Corn Growth and Development. PMR 1009. Iowa State University of Science and Technology, Cooperative Extension Service, Ames, lowa.

Akdemir D and Godfrey OU (2015) EMMREML: Fitting Mixed Models with Known Covariance Structures. Available at: https://cran.r-project.org/package=EMMREML

Akdemir D and Isidro-Sánchez J (2019) Design of training populations for selective phenotyping in genomic prediction. Scientific Reports 9(1446). https://doi.org/https://doi.org/10.1038/s41598-018-38081-6

Auinger H-J, Schönleben M, Lehermeier C, Schmidt M, Korzun V, Geiger HH, Piepho H-P, Gordillo A, Wilde P, Bauer E, and Schön C-C (2016) Model training across multiple breeding cycles significantly improves genomic prediction accuracy in rye (Secale cereale L.). Theoretical and Applied Genetics 129(11): 2043-2053. https://doi.org/10.1007/s00122-016-2756-5

Bajgain P, Zhang X, and Anderson JA (2020) Dominance and GxE interaction effects improve genomic prediction and genetic gain in intermediate wheatgrass (Thinopyrum intermedium). The Plant Genome. John Wiley \& Sons, Ltd 13(1): e20012. https://doi.org/https://doi.org/10.1002/tpg2.20012

Bernal-Vasquez A-M, Möhring J, Schmidt M, Schönleben M, Schön C-C, and Piepho H-P (2014) The importance of phenotypic data analysis for genomic prediction - a case study comparing different spatial models in rye. BMC Genomics 15(1): 646. https://doi.org/10.1186/1471-2164$15-646$

Burgueño J, Campos G de los, Weigel K, and Crossa J (2012) Genomic Prediction of Breeding Values when Modeling Genotype $\times$ Environment Interaction using Pedigree and Dense Molecular Markers. Crop Science 52(2): 707-719. https://doi.org/10.2135/cropsci2011.06.0299

Butler DG, Cullis BR, Gilmour AR, Gogel BJ, and Thompson R (2018) ASReml-R Reference Manual Version 4. VSN International Ltd., Hemel Hempstead

Calus MPL and Vandenplas J (2018) SNPrune: an efficient algorithm to prune large SNP array and sequence datasets based on high linkage disequilibrium. Genetics Selection Evolution 50(1): 34. https://doi.org/10.1186/s12711-018-0404-z

Calus MPL and Veerkamp RF (2011) Accuracy of multi-trait genomic selection using different methods. Genetics Selection Evolution 43(1): 26. https://doi.org/10.1186/1297-9686-43-26

Chang CC, Chow CC, Tellier LC, Vattikuti S, Purcell SM, and Lee JJ (2015) Second-generation PLINK: rising to the challenge of larger and richer datasets. Gigascience 4(7). https://doi.org/10.1186/s13742-015-0047-8

Covarrubias-Pazaran G, Schlautman B, Diaz-Garcia L, Grygleski E, Polashock J, Johnson-Cicalese J, Vorsa N, lorizzo M, and Zalapa J (2018) Multivariate GBLUP Improves Accuracy of Genomic 
Selection for Yield and Fruit Weight in Biparental Populations of Vaccinium macrocarpon Ait. Frontiers in Plant Science 9(1310). https://doi.org/https://doi.org/10.3389/fpls.2018.01310

Da Y, Wang C, Wang S, and Hu G (2014) Mixed Model Methods for Genomic Prediction and Variance Component Estimation of Additive and Dominance Effects Using SNP Markers. PLOS ONE 9(1). https://doi.org/10.1371/journal.pone.0087666

Daetwyler HD, Calus MPL, Pong-Wong R, Campos G de los, and Hickey JM (2013) Genomic Prediction in Animals and Plants: Simulation of Data, Validation, Reporting, and Benchmarking. Genetics 193: 347-365. https://doi.org/10.1534/genetics.112.147983

Dekkers JCM (2007) Prediction of response to marker-assisted and genomic selection using selection index theory. Journal of Animal Breeding and Genetics. John Wiley \& Sons, Ltd 124(6): 331-341. https://doi.org/10.1111/j.1439-0388.2007.00701.x

Erbe M, Pimentel E, Sharifi AR, and Simianer H (2010) Assessment of cross-validation strategies for genomic prediction in cattle. Proceedings of the World Congress on Genetics Applied to Livestock Production Methods an: 553

Falconer DS and Mackay TFC (1996) Introduction to Quantitative Genetics. Longman. Essex Engl.

Hallauer AR, Carena MJ, and Miranda Filho JB (2010) Quantitative genetics in maize breeding. Springer. Berlin

Henderson CR and Quaas RL (1976) Multiple Trait Evaluation Using Relatives' Records. Journal of Animal Science 43(6): 1188-1197. https://doi.org/10.2527/jas1976.4361188x

Hölker AC, Mayer M, Presterl T, Bolduan T, Bauer E, Ordas B, Brauner PC, Ouzunova M, Melchinger AE, and Schön C-C (2019) European maize landraces made accessible for plant breeding and genome-based studies. Theoretical and Applied Genetics 132(12): 3333-3345. https://doi.org/10.1007/s00122-019-03428-8

Hu Z, Li Y, Song X, Han Y, Cai X, Xu S, and Li W (2011) Genomic value prediction for quantitative traits under the epistatic model. BMC Genet 12(15). https://doi.org/https://doi.org/10.1186/1471-2156-12-15

Jia Y and Jannink J-L (2012) Multiple-Trait Genomic Selection Methods Increase Genetic Value $\begin{array}{llllll}\text { Prediction } \quad \text { Accuracy. } & \text { Genetics } & 1513 & \text { LP } & \text { L } & \end{array}$ https://doi.org/10.1534/genetics.112.144246

Jiang Y and Reif JC (2015) Modeling Epistasis in Genomic Selection. Genetics 201(2): 759-768. https://doi.org/10.1534/genetics.115.177907

Lee SH and van der Werf JHJ (2016) MTG2: an efficient algorithm for multivariate linear mixed model analysis based on genomic information. Bioinformatics 32(9): 1420-1422. https://doi.org/10.1093/bioinformatics/btw012

de los Campos G, Vazquez Al, Fernando R, Klimentidis YC, and Sorensen D (2013) Prediction of Complex Human Traits Using the Genomic Best Linear Unbiased Predictor. PLoS Genetics 9(7). 
https://doi.org/https://doi.org/10.1371/journal.pgen.1003608

Lynch M and Walsh B (1998) Genetics and Analysis of Quantitative Traits. Sinauer Associates Mackay TFC (2014) Epistasis and Quantitative Traits: Using Model Organisms to Study Gene-Gene Interactions. Nat Rev Genet. 15(1): 22-33. https://doi.org/10.1038/nrg3627

Martini JWR, Wimmer V, Erbe M, and Simianer H (2016) Epistasis and covariance: how gene interaction translates into genomic relationship. Theoretical and Applied Genetics 129(5): 963976. https://doi.org/10.1007/s00122-016-2675-5

Martini JWR, Gao N, Cardoso DF, Wimmer V, Erbe M, Cantet RJC, and Henner S (2017) Genomic prediction with epistasis models: on the marker-coding-dependent performance of the extended GBLUP and properties of the categorical epistasis model (CE). BMC Bioinformatics 18(3). https://doi.org/https://doi.org/10.1186/s12859-016-1439-1

Meuwissen THE, Hayes BJ, and Goddard ME (2001) Prediction of total genetic value using genome-wide dense marker maps. Genetics 157(4): 1819-1829

Mrode RA (2014) Linear Models for the Prediction of Animal Breeding Values. CABI. https://doi.org/10.1079/9781780643915.0000

Pérez P and de los Campos G (2014) Genome-wide regression and prediction with the BGLR statistical package. Genetics. 2014/07/09. Genetics Society of America 198(2): 483-495. https://doi.org/10.1534/genetics.114.164442

Purcell S, Neale B, Todd-Brown K, Thomas L, Ferreira MAR, Bender D, Maller J, Sklar P, Bakker PIW de, Daly MJ, and Sham PC (2007) PLINK: A Tool Set for Whole-Genome Association and Population-Based Linkage Analyses. American Journal of Human Genetics 81(3): 559-575. https://doi.org/10.1086/519795

Rönnegård L and Shen X (2016) Genomic prediction and estimation of marker interaction effects. bioRxiv 38935. https://doi.org/https://doi.org/10.1101/038935

Runcie D and Cheng H (2019) Pitfalls and Remedies for Cross Validation with Multi-trait Genomic Prediction Methods. G3: Genes/Genomes/Genetics 9(11): 3727 LP - 3741. https://doi.org/10.1534/g3.119.400598

Schlather M (2020) Efficient Calculation of the Genomic Relationship Matrix. bioRxiv. https://doi.org/https://doi.org/10.1101/2020.01.12.903146

Schrag TA, Schipprack W, and Melchinger AE (2019a) Across-years prediction of hybrid performance in maize using genomics. Theoretical and Applied Genetics. Springer Verlag 132(4): 933-946. https://doi.org/10.1007/s00122-018-3249-5

Schrag TA, Schipprack W, and Melchinger AE (2019b) Across-years prediction of hybrid performance in maize using genomics. Theoretical and Applied Genetics 132: 933-946

Stich B and Ingheland D Van (2018) Prospects and Potential Uses of Genomic Prediction of Key 
535 Performance Traits in Tetraploid Potato. Frontiers in Plant Science 9(159).

536 https://doi.org/10.3389/fpls.2018.00159

537 Thompson R and Meyer K (1986) A review of theoretical aspects in the estimation of breeding 538 values for multi-trait selection. Livestock Production Science 15(4): 299-313. 539 https://doi.org/https://doi.org/10.1016/0301-6226(86)90071-0

540 Unterseer S, Author EB, Haberer G, Seidel M, Knaak C, Ouzunova M, Meitinger T, Strom TM, Fries 541 R, Pausch H, Bertani C, Davassi A, Mayer KF, and Schön C-C (2014) A powerful tool for genome 542 analysis in maize: 584 development and evaluation of the high density $600 \mathrm{k}$ SNP genotyping 543 array. BMC Genomics 15(823). https://doi.org/10.1186/1471-2164-15-823

544 VanRaden P (2007) Efficient estimation of breeding values from dense genomic data. Journal of 545 Dairy Science 90: $374-375$

546 VanRaden P (2008) Efficient methods to compute genomic predictions. Journal of Dairy Science 547 91(11): 4414-4423. https://doi.org/10.3168/jds.2007-0980

548 Vojgani E, Pook T, Martini JWR, Hoelker AC, Mayer M, Schoen C-C, and Simianer H (2020) 549 Accounting for epistasis improves genomic prediction of phenotypes with univariate and 550 bivariate models across environments. bioRxiv 2020.10.08.331074. 551 https://doi.org/10.1101/2020.10.08.331074

552 Vojgani E, Pook T, and Simianer H (2019a) EpiGP: Epistatic relationship matrix based genomic 553 prediction of phenotypes. Available at: https://github.com/evojgani/EpiGP

554 Vojgani E, Pook T, and Simianer H (2019b) Phenotype Prediction under Epistasis. in KC, W. (ed.) 555 Epistasis: Methods and Protocols. Springer

556 Wang D, El-Basyoni IS, Baenziger PS, Crossa J, Eskridge KM, and Dweikat I (2012) Prediction of 557 genetic values of quantitative traits with epistatic effects in plant breeding populations. Heredity 558 109(5): 313-319. https://doi.org/10.1038/hdy.2012.44

559 Wang J, Zhou Z, Zhang Zhe, Li H, Liu D, Zhang Q, Bradbury PJ, Buckler ES, and Zhang Zhiwu (2018) 560 Expanding the BLUP alphabet for genomic prediction adaptable to the genetic architectures of 561 complex traits. Heredity 121(6): 648-662. https://doi.org/10.1038/s41437-018-0075-0 